\title{
Efecto del cruzamiento sobre características de crecimiento en ganado cebuíno de la región Chorotega ${ }^{1}$
}

\section{Effect of crossbreeding on growth characteristics in zebu cattle of Chorotega region}

\author{
Mónica Madrigal-Valverde ${ }^{2,3}$, Jorge Camacho-Sandoval ${ }^{4}$, Catalina Salas-Durán ${ }^{5}$
}

1 Recibido: 9 de abril, 2018. Aceptado: 13 de junio, 2018. Este trabajo formó parte de la tesis de Licenciatura en Ingeniería Agronómica con énfasis en Zootecnia, de la primera autora, realizada para la Escuela de Zootecnia, Universidad de Costa Rica, San José, Costa Rica.

2 Universidade Federal da Bahia, Escola de Medicina Veterinária e Zootecnia, Salvador, Bahía, Brasil. madrigalvalverdem@gmail.com (autor para correspondencia; https://orcid.org/0000-0002-4688-0627).

3 Instituto Tecnológico de Costa Rica, Centro de Investigación y Desarrollo en Agricultura Sostenible para el Trópico Húmedo, Sede San Carlos, Alajuela, Costa Rica.

4 Servicios Estadísticos Camacho \& Fernández S.A. Alajuela, Costa Rica. jorge.camacho.s@gmail.com

5 Universidad de Costa Rica, Escuela de Zootecnia, Campus Rodrigo Facio, San Pedro, San José, Costa Rica. catalina.salas@ucr.ac.cr (https:// orcid.org/0000-0001-5627-6346).

\section{Resumen}

Introducción. Las investigaciones sobre el efecto del cruzamiento entre Bos indicus * Bos indicus son escasos, no obstante, el cruzamiento es una herramienta de optimización importante para el productor pecuario. Objetivo. El objetivo de este trabajo consistió en determinar el comportamiento de las características de crecimiento con base en diferentes proporciones de cruzamientos raciales Bos indicus. Materiales y métodos. El análisis se realizó con base en registros productivos de una ganadería con bovinos seleccionados Brahman y Nelore, y ganado comercial, ubicada en la región Pacífico Norte, Costa Rica. Se utilizaron los registros de 4294 animales, recopilados durante el periodo 20022012. Los grupos raciales incluidos en el estudio fueron: Brahman, Nelore, Gucerá, $1 / 2$ Brahman $1 / 2$ Nelore, $1 / 2$ Brahman $1 / 2$ Gucerá, 1/4 Brahman 3/4 Nelore y 1/4 Nelore 3/4 Brahman. Se evaluaron las características de peso al nacimiento, peso al destete, ganancia diaria de peso al destete, peso al año de edad, ganancia diaria de peso al año de edad, peso a los 18 meses, ganancia diaria de peso a los 18 meses, según grupo racial de la cría y de los progenitores. Adicionalmente, se analizaron los efectos no aditivos (sexo, época y año de nacimiento) y su influencia sobre las características. Resultados. Los grupos Brahman, Nelore y 1/4 Nelore 3/4 Brahman tuvieron los mayores pesos en todas las edades, los animales machos presentaron pesos superiores para la mayor parte de las características analizadas $(\mathrm{P}<0,05)$; los animales nacidos en época lluviosa presentaron los mayores pesos para todas las características, respecto a la época seca, mientras que el año de nacimiento no presentó tendencias. La raza de la madre influyó en edades tempranas de la cría y el grupo racial del padre en las diferencias posteriores al año de edad. Además, las variables sexo y época de nacimiento presentaron comportamientos definidos, mientras el año de nacimiento mostró resultados fluctuantes. Conclusión. En cruzamientos raciales Bos indicus, el grupo racial de los progenitores influencia las características de crecimiento de la progenie.

Palabras clave: Bos indicus, ganancia de peso, mejoramiento animal, producción pecuaria.

\begin{abstract}
Introduction. There is little research about the effect of crossbred between Bos indicus * Bos indicus breeding, however, crossbreeding is an important optimization's tool used by the livestock producers. Objective. The objective


of this study was to determine the behavior of growth characteristics on different proportions of Bos indicus breed crosses. Materials and methods. The analysis was made based on production records of a selected cattle farm with Brahman, Nelore and commercial stock located in the North Pacific region, Costa Rica. The records of 4.294 animals, collected during the 2002-2012 period in the farm, were used; the breed groups included in the study were: Brahman, Nelore, Guzerat, 1/2 Brahman 1/2 Nelore, 1/2 Brahman 1/2 Guzerat, 1/4 Brahman 3/4 Nelore, and 1/4 Nelore 3/4 Brahman. Birth weight, weaning weight, weaning daily weight gain, year old weight, year old daily weight gain, 18 months weight, and 18 months daily weight gain were analyzed according to the breed group of the offspring and the parents. In addition, the non-additive effects (sex, season of birth and year of birth) and their influence on the characteristics were analyzed. Results. It was found that the Brahman, Nelore and 1/4 Nelore 3/4 Brahman breed groups had the highest weights at all ages, male animals presented higher weights for most of the analyzed characteristics $(\mathrm{P}<0.05)$; the animals born in the rainy season presented the highest weights for all the characteristics, in relation to the dry season, while the year of birth did not present trends. The study concluded that the breed group of the mother influenced the early ages of the offspring and the breed group of the father caused differences after one year of age. Likewise, the study concluded that the variables sex and season of birth presented defined behaviors, while the year of birth have fluctuating results. Conclusion. In crossbreds Bos indicus, the breed group of the progenitors have influences in the growth characteristics of progeny.

Keywords: Bos indicus, weight gain, animal breeding, livestock production.

\section{Introducción}

La economía alimentaria mundial está siendo cada vez más impulsada por el cambio de las dietas hacia productos de origen animal como la carne, la leche y los productos lácteos (FAO, 2015). América Latina y el Caribe representan aproximadamente el 14\% de los inventarios mundiales de las principales especies de ganado (FAO, 2014), siendo una región de importancia en la producción pecuaria. En Costa Rica, la producción de alimentos de origen vacuno se concentra en 37171 fincas, distribuidas en 80 de los 82 cantones del país, para un total de 1278817 cabezas de ganado reportadas en el 2014; en la provincia de Guanacaste se concentra el 18\% del total de fincas que albergan sistemas de producción de bovinos de carne (INEC, 2014).

A nivel de campo, el sistema de cruzamiento es una de las herramientas que posee el criador de ganado comercial, para ejecutar el mejoramiento genético en los hatos, el cual permite mejorar características de crecimiento animal (Newman et al., 2002; Teixeira y Albuquerque, 2005; Perotto et al., 2006; Martínez-González et al., 2008; Ramírez, 2009; Vargas-Leitón y Romero-Zúñiga, 2010; Rodrigues et al., 2014; Schiermiester et al., 2015; Côrtes-Carvalho et al., 2017; Dal-Pizzol et al., 2017). Los estudios de cruzamientos entre razas Bos taurus x Bos indicus (Flôres et al., 2004; Perotto et al., 2006; McManus et al., 2008; Lopes et al., 2010; Marcondes et al., 2012; Pitombo et al., 2013; Dillon et al., 2015; Campbell et al., 2016; Côrtes-Carvalho et al., 2017), indican mejoría en las características estudiadas en cada caso particular. No obstante, las investigaciones en cruzamiento entre ganado cebú son escasas (Cubas et al., 2001; de-Azambuja-Ribeiro et al., 2008; Pitombo et al., 2013). El objetivo de este trabajo consistió en determinar el comportamiento de las características de crecimiento en diferentes proporciones de cruzamientos raciales Bos indicus.

\section{Materiales y métodos}

\section{Ubicación del estudio y animales}

La ganadería en donde se realizó el estudio se encuentra en la región Chorotega, Costa Rica, a 86 msnm, con temperaturas que varían entre $22,1^{\circ} \mathrm{C}$ y $33,0{ }^{\circ} \mathrm{C}$, y un régimen de lluvias de $1517 \mathrm{~mm} /$ año (IMN, 2008). La 
topografía de la ganadería se constató como $90 \%$ plana y $10 \%$ semiplano. La orientación productiva de la finca es la venta de animales como reproductores de los grupos raciales Brahman, Nelore y animales cruzados con fines comerciales. En general, se mantiene una alimentación basada en forraje y sales minerales, con la excepción de las crías, a las que se les proporciona 1-2 kg de alimento balanceado un mes posterior al destete. El manejo del pastoreo es en sistema tipo pastel, con una rotación basada en la disponibilidad de pasto, y los repastos tienen una cobertura mayoritaria de mombaza (Pannicum Maximun cv. Mombaza), en menor proporción braquipará (Brachiaria arrecta), toledo (Brachiaria Brizantha cv Toledo), alemán (Echynochloa polystachya), además de árboles dispersos.

En el hato total se mantienen dos sistemas de cruzamientos, uno para las líneas seleccionadas y otro para los animales comerciales. En el sistema de líneas seleccionadas se mantienen padres y madres de los grupos Brahman y Nelore, mientras que en el sistema de animales comerciales se incorporan madres Brahman, Nelore y $1 / 2$ Brahman $1 / 2$ Nelore, y los padres corresponden a Brahman, Nelore y Gucerá.

En la finca se realiza el manejo zootécnico necesario (cuidado sanitario, instalaciones, identificación de animales, pesajes, direccionamiento de la reproducción en esquema de cruzamiento), así como la incorporación de datos a la base de registros, en un software computacional diseñado para contener registros zootécnicos.

\section{Colección y ordenamiento de datos}

La base de datos del software computacional, incluía datos productivos del hato (identificación del animal, de los progenitores y el peso en kilogramos registrado para cada año), organizados por periodos de diez años. Para el estudio, se utilizaron los datos correspondientes al periodo 2002-2012.

La identificación del animal incluyó datos de sexo de la cría y fecha de nacimiento, con lo que se estableció la época y año de nacimiento.

Se ajustaron los datos según las fórmulas de Beef Improvement Federation (2010), para la generación de nuevas variables: peso ajustado a 205 días (PD), ganancia diaria de peso de nacimiento a destete (GDPND), peso ajustado a 365 días (PA: peso ajustado al año de edad), ganancia diaria de peso de destete al año (GDPA), peso ajustado a 550 días (P18: peso ajustado a los 18 meses de edad) y ganancia diaria de peso del año a 18 meses (GDP18). En el Cuadro 1, se describen los niveles y variables analizadas.

Cuadro 1. Variables explicativas para los modelos de análisis para determinar el comportamiento de las características de crecimiento en diferentes grados de cruzamientos raciales Bos indicus. Costa Rica, 2012.

Table 1. Explanatory variables for analysis models to determine the behavior of growth characteristics in different racial grades of Bos indicus crossbreds. Costa Rica, 2012.

\begin{tabular}{lcl}
\hline Variable & Niveles & Valores \\
\hline Año de nacimiento & 11 & $2001,2002,2003,2004,2005,2006,2007,2008,2009,2010,2011$ \\
Época de nacimiento & 2 & Seca, lluviosa \\
Sexo & 2 & Hembra, macho \\
Grupo racial de cría & 6 & $\begin{array}{l}1 / 2 \text { Brahman } 1 / 2 \text { Gucerá, 1/2 Brahman 1/2 Nelore, } 1 / 4 \text { Brahman } 3 / 4 \text { Nelore, } \\
1 / 4 \text { Nelore } 3 / 4 \text { Brahman, Brahman, Nelore }\end{array}$ \\
Grupo racial de madre & 3 & $1 / 2$ Brahman $1 / 2$ Nelore, Brahman, Nelore \\
Grupo racial de padre & 3 & Brahman, Nelore, Gucerá \\
\hline
\end{tabular}




\section{Análisis estadístico}

El estudio de los registros se realizó por medio de dos programas computacionales para la corrección de datos en la base de matriz, por lo tanto, el estudio se realizó en dos etapas. En la primera se estimaron las medias ( \pm error estándar), así como análisis de componentes de varianza para las características productivas. En la segunda etapa se ajustaron distintos modelos para las variables de interés, que incluyeron las principales fuentes de variación identificadas, así como covariables.

Para cada una de las variables de interés se ajustó un modelo mixto, mediante el procedimiento "Mixed" del programa estadístico SAS versión 9.2 (SAS Institute, 2009).

Modelo general

$y_{i j k}=\mu+G C_{i}+G R_{j}+\varepsilon_{i j k}$

En donde:

$y_{i j k}$ : valor de la variable de interés para el k-ésimo individuo del j-ésimo grupo racial y el i-ésimo grupo contemporáneo.

$\mu$ : efecto común a todos los individuos.

$G C_{i}$ : efecto aleatorio del i- ésimo del grupo contemporáneo, formado por la combinación de sexo, año y época de nacimiento.

$G R_{j}$ : efecto fijo del j-ésimo del grupo racial de la cría.

$\varepsilon_{i j k}:$ error residual aleatorio.

Para las características evaluadas después del destete, se utillizó un modelo Anova con covariables. Para la estimación, el modelo involucró solamente la raza de la cría e incluyó los factores: año de nacimiento, época de nacimiento, sexo, raza de la cría y, como covariable el peso al destete.

Para estudiar el efecto de los factores incluidos en los grupos contemporáneos de forma independiente, se ajustó un modelo que incluyó además del efecto del grupo racial, el efecto de año, época de nacimiento y sexo, considerándolos como efectos fijos. El modelo se estimó con base en el procedimiento GLM (modelos lineales generalizados).

Con el propósito de estudiar el efecto del grupo racial de la madre y del padre, se realizó un ANOVA que incluyó los efectos de las razas de progenitores y los factores: año de nacimiento, época de nacimiento, sexo, grupo racial de la madre y grupo racial del padre, debido a que, el sistema de ecuaciones que incluyen grupos raciales de crías y padres no pueden ser resueltos juntos.

El modelo empleado fue el siguiente:

$$
y_{i j k l m}=\mu+G C_{i}+G R_{j}+G R M_{k}+G R P_{l}+\varepsilon_{i j k l m}
$$

En donde:

$y_{i j k l m}$ : valor de la variable de interés para el m-ésimo individuo, del l-ésimo grupo racial del padre, del k-ésimo grupo racial de la madre, del j-ésimo del grupo racial de la cría y el i-ésimo grupo contemporáneo.

$\mu$ : efecto común a todos los individuos. 
$G C_{i}$ : efecto aleatorio del i-ésimo del grupo contemporáneo, formado por la combinación de sexo, año y época de nacimiento.

$G R_{j}$ : efecto fijo del j-ésimo del grupo racial de la cría.

$G R M_{k}$ : efecto del k-ésimo grupo racial de la madre.

$G R P_{l}$ : efecto del l-ésimo grupo racial del padre.

$\varepsilon_{i j k l m}$ : error residual aleatorio.

Se estimó el medio mínimo cuadrático de los grupos raciales para todas las variables estudiadas, así como las diferencias estadísticas entre ellas, mediante la prueba de Tukey-Kramer con nivel de significancia de $\mathrm{P}<0,05$. Para la primera etapa de los análisis estadísticos se utilizó el software SPSS (SPSS, 2012).

\section{Resultados}

\section{Efectos no aditivos}

\section{Año de nacimiento}

La comparación de medias entre las características productivas y la variable año de nacimiento de la cría, señaló un comportamiento fluctuante de los valores para cada característica, sin efecto significativo ( $\mathrm{P}>0,05)$.

\section{Época de nacimiento}

Para la característica productiva de peso al nacimiento y peso ajustado a los 18 meses, los animales nacidos durante la época lluviosa fueron más pesados con respecto a los nacidos en época seca $(\mathrm{P}<0,05)($ Cuadro 2$)$.

Cuadro 2. Medias de peso de las crías de la ganadería con bovinos seleccionados Brahman y Nelore, y ganado comercial, al nacimiento, a los 205 días (destete), 365 días (al año de edad) y 550 días (18 meses de edad) y ganancias diarias de peso, según época de nacimiento de la cría. Región Pacífico Norte, Costa Rica. Periodo 2002-2012.

Table 2. Weight means of the calves of selected cattle Brahman and Nelore farm and commercial livestock, at birth, 205 days (weaning), 365 days (at year for age), and 550 days (18 months for age) and daily weight gain, according to the breeding season. North Pacific Region, Costa Rica. Period 2002-2012.

\begin{tabular}{|c|c|c|c|c|c|c|c|c|c|c|c|}
\hline \multirow[b]{2}{*}{ Época } & \multicolumn{2}{|c|}{ Nacimiento } & \multicolumn{3}{|c|}{ Destete } & \multicolumn{3}{|c|}{ Año } & \multicolumn{3}{|c|}{18 meses } \\
\hline & $\mathbf{N}$ & $\begin{array}{c}\text { Peso } \\
\text { (kg) }\end{array}$ & $\mathbf{N}$ & $\begin{array}{c}\text { Peso Aj. } \\
\text { (kg) }\end{array}$ & $\begin{array}{l}\text { GDP } \\
\text { (g/d) }\end{array}$ & $\mathbf{N}$ & $\begin{array}{l}\text { Peso Aj. } \\
\text { (kg) }\end{array}$ & $\begin{array}{l}\text { GDP } \\
(\mathrm{g} / \mathrm{d})\end{array}$ & $\mathbf{N}$ & $\begin{array}{c}\text { Peso Aj. } \\
\text { (kg) }\end{array}$ & $\begin{array}{l}\text { GDP } \\
\text { (g/d) }\end{array}$ \\
\hline Lluviosa & 1838 & $32,39^{\mathrm{a}}$ & 1023 & $188,24^{\mathrm{a}}$ & $761^{\mathrm{a}}$ & 1180 & $223,59^{a}$ & $260^{a}$ & 441 & $341,34^{\mathrm{a}}$ & $357^{\mathrm{a}}$ \\
\hline Seca & 2456 & $32,08^{\mathrm{b}}$ & 1810 & $187,96^{\mathrm{a}}$ & $760^{\mathrm{a}}$ & 764 & $217,94^{\mathrm{a}}$ & $225^{\mathrm{a}}$ & 873 & $290,02^{\mathrm{b}}$ & $258^{\mathrm{a}}$ \\
\hline
\end{tabular}

N: número de observaciones, Peso Aj: peso ajustado, GDP: ganancia diaria de peso, g/d: gramos por día / N: number of observations per group, Peso Aj: Adjusted weight, GDP: daily weight gain, g/d: grams per day.

Letras con superíndices diferentes dentro de columna indican diferencias significativas $(\mathrm{P}<0,05) /$ Different letters in the same column indicate significant differences $(\mathrm{P}<0.05)$. 
Sexo de la cría

Los animales machos resultaron más pesados en comparación a las hembras desde el nacimiento al año de edad $(\mathrm{P}<0,05)$ (Cuadro 3), excepto para la variable peso ajustado a los 550 días.

Cuadro 3. Medias de peso de las crías de la ganadería con bovinos seleccionados Brahman y Nelore y ganado comercial, en diferentes etapas de crecimiento según sexo de la cría. Región Pacífico Norte, Costa Rica. Periodo 2002-2012.

Table 3. Weight means of the calves of the selected cattle in the Brahman, Nelore and commercial livestock farm, at different stages of growth according to the sex of the calf . North Pacific Region, Costa Rica. Period 2002-2012.

\begin{tabular}{|c|c|c|c|c|c|c|c|c|c|c|c|}
\hline \multirow[b]{2}{*}{ Sexo } & \multicolumn{2}{|c|}{ Nacimiento } & \multicolumn{3}{|c|}{ Destete } & \multicolumn{3}{|c|}{ Año } & \multicolumn{3}{|c|}{18 meses } \\
\hline & $\mathbf{N}$ & $\begin{array}{c}\text { Peso } \\
(\mathrm{kg})\end{array}$ & $\mathbf{N}$ & $\begin{array}{l}\text { Peso Aj. } \\
\text { (kg) }\end{array}$ & $\begin{array}{l}\text { GDP } \\
\text { (g/d) }\end{array}$ & $\mathbf{N}$ & $\begin{array}{l}\text { Peso Aj. } \\
\quad(k g)\end{array}$ & $\begin{array}{l}\text { GDP } \\
\text { (g/d) }\end{array}$ & $\mathbf{N}$ & $\begin{array}{l}\text { Peso Aj. } \\
\text { (kg) }\end{array}$ & $\begin{array}{l}\text { GDP } \\
(\mathrm{g} / \mathrm{d})\end{array}$ \\
\hline Hembra & 2134 & $31,68^{\mathrm{a}}$ & 1478 & $181,88^{\mathrm{a}}$ & 733,46 & 961 & $210,23^{a}$ & $176,40^{\mathrm{a}}$ & 676 & $319,03^{\mathrm{a}}$ & $331,60^{\mathrm{a}}$ \\
\hline Macho & 2160 & $32,79^{b}$ & 1355 & $194,31^{\mathrm{b}}$ & 788,18 & 983 & $231,30^{\mathrm{b}}$ & $308,12^{\mathrm{b}}$ & 638 & $312,34^{\mathrm{a}}$ & $282,67^{\mathrm{a}}$ \\
\hline
\end{tabular}

$\mathrm{N}$ : número de observaciones por grupo, Peso Aj: peso ajustado, GDP: ganancia diaria de peso, g/d: gramos por día / N: number of observations per group, Peso Aj: adjusted weight, GDP: daily weight gain. g/d: grams per day.

Letras con superíndices entre columnas indican diferencias significativas $(\mathrm{P}<0,05) /$ Different letters in the same column indicate significant differences $(\mathrm{P}<0.05)$.

\section{Efecto del grupo racial de la cría y progenitores}

Para la característica de peso al nacimiento $(\mathrm{PN})$, según grupo racial de la cría, el grupo racial Brahman presentó diferencias $(\mathrm{P}<0,05)$ con los $1 \frac{1}{2}$ Brahman 1/2 Nelore, 1/4 Brahman 3/4 Nelore y Nelore (Cuadro 4).

Las hembras de la raza Brahman presentaron crías con mayor peso al nacimiento $(\mathrm{P}<0,05)$, mientras que, el genotipo de 50\% Brahman 50\% Nelore obtuvo descendientes con pesos menores al nacimiento $(\mathrm{P}<0,05)$, en relación con aquellos con madres 100\% Brahman o 100\% Nelore (Cuadro 5). Además, los padres de la raza Gucerá presentaron los hijos con mayor peso al nacimiento $(\mathrm{P}<0,05)$, con respecto a los otros padres (Cuadro 6).

Para la característica de peso al destete (PD), no hubo diferencias $(\mathrm{P}>0,05)$ entre los grupos Brahman y Nelore. La progenie de las hembras Nelore presentó mayores pesos al destete $(\mathrm{P}<0,05)$ que la progenie de las madres cuya composición genética fue 100\% Brahman y 50\% Brahman 50\% Nelore. No hubo diferencias $(\mathrm{P}>0,05)$ en el PD de la progenie entre padres Brahman y Nelore, pero estos grupos conjuntamente sí difirieron $(\mathrm{P}<0,05)$ de la progenie de machos Gucerá.

Al observar la ganancia diaria de peso del nacimiento al destete (GDPD), según el grupo racial de las crías, no se encontraron diferencias $(\mathrm{P}>0,05)$ entre los grupos Brahman y Nelore. Con respecto a la característica de GDPD según el grupo racial de los progenitores, no se encontraron diferencias $(\mathrm{P}>0,05)$ entre los grupos Brahman y Nelore. No hubo diferencias $(\mathrm{P}>0,05)$ en el peso al año entre los diferentes grupos raciales conformados. Tampoco hubo efecto del genotipo materno sobre PA y GDPA.

Respecto al comportamiento de las características PA y GDPA, según grupo racial del padre, no hubo diferencia entre los grupos Brahman y Nelore $(\mathrm{P}>0,05)$, estos grupos conjuntamente fueron diferentes $(\mathrm{P}<0,05)$ del Gucerá. No hubo diferencias $(\mathrm{P}>0,05)$ para la característica $\mathrm{P} 18$ entre los grupos raciales de la cría ni de los progenitores. Para la característica GDP18 no hubo diferencias $(\mathrm{P}>0,05)$ para el grupo racial de la cría ni el grupo racial de la madre. Por el contrario, sí hubo diferencias con significado estadístico $(\mathrm{P}<0,05)$ entre los grupos de padres. 


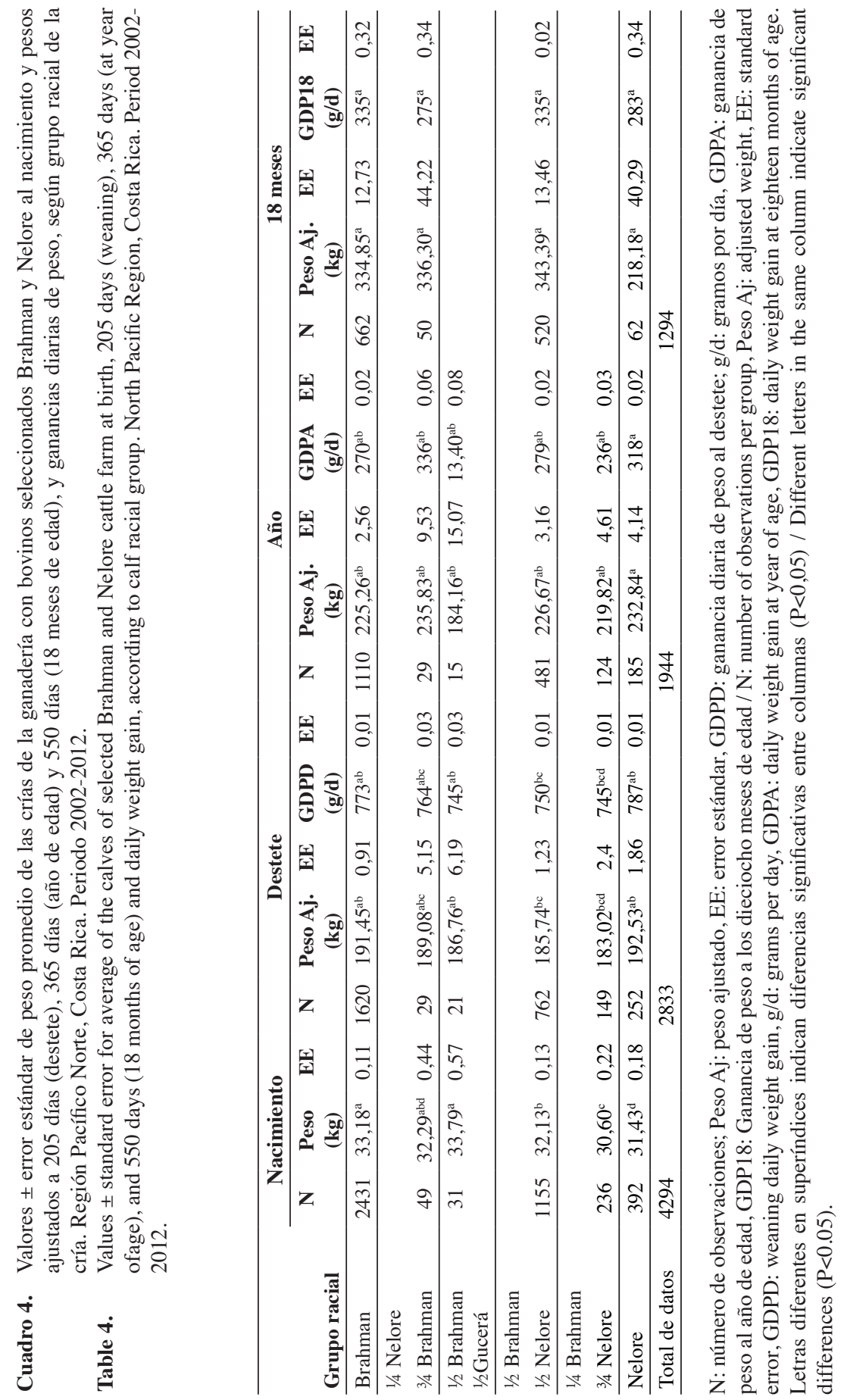


Cuadro 5. Valores de peso promedio de las crías de la ganadería con bovinos seleccionados Brahman y Nelore al nacimiento, a los 205 días (destete), 365 días (al año de edad) y 550 días (18 meses de edad) y ganancias diarias de peso y ganancias diarias de peso, según grupo racial del madre. Región Pacífico Norte, Costa Rica. Periodo 2002-2012.

Table 5. Average weight values of the calves from the selected Brahman and Nelore cattle farm at birth, 205 days (weaning), 365 days (at year of age), and 550 days (18 months of age) and daily weight gain, by maternal racial group. North Pacific Region, Costa Rica. Period 2002-2012.

\begin{tabular}{|c|c|c|c|c|c|c|c|c|c|c|c|}
\hline \multirow[b]{2}{*}{$\begin{array}{l}\text { Grupo } \\
\text { Racial }\end{array}$} & \multicolumn{2}{|c|}{ Nacimiento } & \multicolumn{3}{|c|}{ Destete } & \multicolumn{3}{|c|}{ Año } & \multicolumn{3}{|c|}{18 meses } \\
\hline & $\mathbf{N}$ & $\begin{array}{l}\text { Peso } \\
\text { (kg) }\end{array}$ & $\mathbf{N}$ & $\begin{array}{l}\text { Peso Aj. } \\
\text { (kg) }\end{array}$ & $\begin{array}{l}\text { GDP } \\
\text { (g/d) }\end{array}$ & $\mathbf{N}$ & $\begin{array}{l}\text { Peso Aj. } \\
\text { (kg) }\end{array}$ & $\begin{array}{c}\text { GDP } \\
(\mathrm{kg} / \mathrm{d})\end{array}$ & $\mathbf{N}$ & $\begin{array}{l}\text { Peso Aj. } \\
\text { (kg) }\end{array}$ & $\begin{array}{l}\text { GDP } \\
\text { (g/d) }\end{array}$ \\
\hline $\begin{array}{l}1 / 2 \text { Brahman } \\
1 / 2 \text { Nelore }\end{array}$ & 2511 & $31,60^{\mathrm{a}}$ & 178 & $185,31^{\mathrm{a}}$ & $750^{\mathrm{a}}$ & 153 & $208,15^{\mathrm{a}}$ & $163^{\mathrm{a}}$ & 66 & $339,69^{\mathrm{a}}$ & $291^{a}$ \\
\hline Brahman & 1721 & $33,04^{b}$ & 2398 & $187,99^{\mathrm{a}}$ & $756^{\mathrm{a}}$ & 1603 & $211,81^{\mathrm{a}}$ & $186^{\mathrm{a}}$ & 1063 & $336,16^{\mathrm{a}}$ & $319^{\mathrm{a}}$ \\
\hline Nelore & 31 & $32,21^{\mathrm{c}}$ & 257 & $194,56^{\mathrm{b}}$ & $792^{\mathrm{b}}$ & 188 & $218,65^{\mathrm{a}}$ & $229^{\mathrm{a}}$ & 185 & $357,65^{\mathrm{a}}$ & $433 a^{b}$ \\
\hline Total de datos & 4263 & & 2833 & & & 1944 & & & 1314 & & \\
\hline
\end{tabular}

$\mathrm{N}$ : número de observaciones, Peso Aj: peso ajustado, GDP: ganancia diaria de peso / N: number of observations per group, Peso Aj: adjusted weight, GDP: daily weight gain.

Letras diferentes en superíndices indican diferencias significativas entre columnas $(\mathrm{P}<0,05) /$ Different letters in the same column indicate significant differences $(\mathrm{P}<0.05)$.

Cuadro 6. Valores de peso promedio de las crías al nacimiento de la ganadería con bovinos seleccionados Brahman y Nelore, al nacimiento, a los 205 días (destete), 365 días (al año de edad) y 550 días (18 meses de edad) y ganancias diarias de peso y ganancias diarias de peso, según grupo racial del padre. Región Pacífico Norte, Costa Rica. Periodo 2002-2012.

Table 6. Average weight values of the calves from the selected Brahman and Nelore cattle farm at birth, 205 days (weaning), 365 days (at year of age), and 550 days (18 months of age) and daily weight gain, by paternal racial group. North Pacific Region, Costa Rica. Period 2002-2012.

\begin{tabular}{|c|c|c|c|c|c|c|c|c|c|c|c|}
\hline \multirow[b]{2}{*}{$\begin{array}{l}\text { Grupo } \\
\text { Racial }\end{array}$} & \multicolumn{2}{|c|}{ Nacimiento } & \multicolumn{3}{|c|}{ Destete } & \multicolumn{3}{|c|}{ Año } & \multicolumn{3}{|c|}{18 meses } \\
\hline & $\mathbf{N}$ & $\begin{array}{c}\text { Peso } \\
(\mathrm{kg})\end{array}$ & $\mathbf{N}$ & $\begin{array}{c}\text { Peso Aj. } \\
(\mathrm{kg})\end{array}$ & $\begin{array}{l}\text { GDP } \\
(\mathrm{g} / \mathrm{d})\end{array}$ & $\mathbf{N}$ & $\begin{array}{c}\text { Peso Aj. } \\
\text { (kg) }\end{array}$ & $\begin{array}{l}\text { GDP } \\
(\mathrm{g} / \mathrm{d})\end{array}$ & $\mathbf{N}$ & $\begin{array}{c}\text { Peso Aj. } \\
(\mathrm{kg})\end{array}$ & $\begin{array}{l}\text { GDP } \\
(\mathrm{g} / \mathrm{d})\end{array}$ \\
\hline Brahman & 2511 & $32,41^{\mathrm{a}}$ & 1654 & $192,72^{\mathrm{a}}$ & $783^{\mathrm{a}}$ & 1142 & $226,64^{\mathrm{a}}$ & $279^{a}$ & 835 & $353,55^{\mathrm{a}}$ & $385^{\mathrm{a}}$ \\
\hline Gucerá & 31 & $33,02^{\mathrm{b}}$ & 21 & $188,04^{\mathrm{b}}$ & $761^{\mathrm{b}}$ & 15 & $185,12^{\mathrm{b}}$ & $19,48 \mathrm{a}$ & & & \\
\hline Nelore & 1752 & $31,41^{\mathrm{a}}$ & 1158 & $187,10^{\mathrm{a}}$ & $755^{\mathrm{a}}$ & 787 & $226,85^{\mathrm{a}}$ & $280 \mathrm{~b}$ & 475 & $325,68^{a}$ & $310^{\mathrm{b}}$ \\
\hline Total de datos & 4294 & & 2833 & & & 1944 & & & 1314 & & \\
\hline
\end{tabular}

N: número de observaciones, Peso Aj: peso ajustado, GDP: ganancia diaria de peso / N: number of observations per group, Peso Aj: Adjusted weight, GDP: daily weight gain.

Letras diferentes en superíndices indican diferencias significativas entre columnas $(\mathrm{P}<0,05) /$ Different letters in the same column indicate significant differences $(\mathrm{P}<0.05)$

\section{Discusión}

Para el efecto no aditivo de año de nacimiento no fue observada ninguna tendencia, pero, el año de nacimiento es un efecto ambiental externo al animal que puede influir en el desarrollo del mismo (Rocha-Sarmento et al., 2003). En investigaciones anteriores (Cubas et al., 2001; Martínez-González et al., 2008), se han reportado diferencias significativas $(\mathrm{P} \leq 0,05)$ en el desarrollo de los animales, debidas al año de nacimiento del ternero, esto por la variación en los factores edafoclimáticos entre años, que afectan la disponibilidad de alimento para la madre 
y la cría (Cubas et al., 2001). Adicionalmente, estas variaciones pueden ser específicas de cada región, lo que puede contribuir directa o indirectamente para que existan esas diferencias (Rocha-Sarmento et al., 2003).

Un peso mayor presentado por los animales nacidos en invierno coincide con lo encontrado en la literatura (Cubas et al., 2001; García-Henao et al., 2003; Martínez-González et al., 2008). El desempeño diferenciado por mes de nacimiento del ternero es conocido, aunque no se encuentran explicaciones biológicas del fenómeno en la literatura. No obstante, este comportamiento puede tener relación con variaciones en las cargas parasitarias e infecciosas, la capacidad de carga en cada estación y las reservas corporales de la madre, consecuentes a cada época del año (García-Henao et al., 2003).

En el presente estudio los mayores pesos fueron registrados por animales nacidos durante la época lluviosa. Esto se puede deber a que, en esa época el pasto es abundante y provee de más alimento a las crías, a las madres preñadas y lactantes. Cuando es época seca en la zona de estudio el alimento escasea, debido a las pocas o inexistentes lluvias, lo cual provoca baja ganancia de peso y bajos pesos. La anterior hipótesis es apoyada por los datos de la estación meteorológica cercana, propiedad del Instituto Meteorológico Nacional de Costa Rica. Esta registra, históricamente, una precipitación media al mes de 44,5 mm con 3,5 días de lluvia durante el verano, mientras que, en la época lluviosa, la media de precipitación alcanza hasta 240,2 mm con 17,5 días de lluvia al mes (IMN, 2008). El mes de nacimiento es una característica que puede ser manejada por el propietario a través de monta controlada o inseminación artificial a tiempo fijo, para mejorar sus índices productivos y responder a la demanda del mercado.

Los resultados de la variable de sexo de las crías coincidieron con otros estudios (Cubas et al., 2001; GarcíaHenao et al., 2003; Jenkins y Ferrell, 2004; Rodrigues-Paulino et al., 2008; Toral et al., 2011), donde los animales machos Bos indicus presentaron mayores pesos en comparación con los animales hembra. Existe un dimorfismo sexual en este grupo, el cual hace que los animales machos sean superiores en peso, en comparación con los animales hembra (Cubas et al. 2001).

Las diferencias de peso entre macho y hembra se encontraron desde el nacimiento y hasta el año de edad. En la raza Nelore con edades mayores al año, la hembra tiene una mayor capacidad de consumo, lo cual repercute en un mayor peso en comparación con los machos (Rodrigues-Paulino et al., 2008). Este comportamiento también fue observado en el presente estudio.

Para la discusión de las características según grupo racial de la cría, no se encontraron estudios con la totalidad de grupos raciales incluidos en la presente investigación, por lo tanto, se realizó una comparación con investigaciones donde se incluyeron una o más grupos raciales coincidentes.

Para la característica peso al nacimiento (PN), los individuos que reportaron el mayor peso fueron del grupo racial Gucerá, esta diferencia puede deberse a que el grupo de padres de esta raza fue el que presentó el menor número de datos de progenie.

El PN de bovinos Nelore ha sido reportado con valores menores en otras investigaciones (Cubas et al., 2001; Lopes et al., 2010). A su vez, no se han presentado diferencias significativas ( $>>0,05)$ entre el grupo Nelore y animales $1 / 2$ Nelore $1 / 2$ Bos indicus, siendo el grupo cruzado el de mayor peso al nacer (Cubas et al., 2001). Estas diferencias de peso pueden deberse a diferencias de manejo y ambiente entre la hacienda de este estudio y las condiciones en las haciendas estudiadas por otros autores.

La característica PD se encuentra relacionada significativamente con el peso al nacer $(\mathrm{P}>0,05)$ (MartínezGonzález et al., 2008); no obstante, debe contemplarse el efecto materno que influencia la magnitud del PD. La influencia del grupo racial de los progenitores sobre las características al destete es explicado como influencia materna, debido a la dependencia del ternero de la madre para su cuidado y alimentación (Rocha-Sarmento et al., 2003).

El PD para animales Nelore varía entre 147 y 196,72 kg (Cubas et al., 2001; Del Valle-Garnero et al., 2001; Vieira et al., 2006; Boligon et al., 2010; Lopes et al., 2010; Gonçalves et al., 2011), rango en el que se encuadra el desempeño del grupo estudiado. 
En cuanto a la ganancia diaria de peso del nacimiento al destete (GDPD), se han reportado en literatura valores inferiores a los obtenidos en el presente estudio (Rocha-Sarmento et al., 2003; Vieira et al., 2006; Lopes et al., 2010), inclusive, Teixera y Albuquerque (2005) establecieron que el valor medio de ganancia de peso al destete de la población Nelore analizada fue de 659 gramos/día. Este valor es 128 gramos por debajo de los encontrados, y es posible que las diferencias se deban al tamaño de muestra de la investigación $(n=252)$.

Algunos autores indican que los individuos Brahman cuentan con pesos al destete y ganancias de peso inferiores a los que arrojan los resultados de este estudio (Lunstra y Cundiff, 2003; Jenkins y Ferrell, 2004; Lopes et al., 2010). A su vez se reportan PD y GDPD menores para animales de padres Gucerá (Toral et al., 2011). Sin embargo, ninguno de los trabajos consultados fue desarrollado en la región centroamericana, por lo que las diferencias en peso pueden deberse a un efecto ambiental.

Para PA y GDPA, los grupos Nelore y Brahman no fueron significativamente diferentes al grupo de mayor peso ( $\mathrm{P}>0,05)$; los animales Nelore registran en literatura valores cercanos a lo encontrado en el presente estudio $(231 \mathrm{~g} \pm 114 \mathrm{~kg} ; 233,05 \mathrm{~kg})$ (Rocha-Sarmento et al., 2003; Boligon et al., 2010), o GDPA superiores (245,09 g; 258,0 $\mathrm{g} \pm 3$,6) (Gonçalves et al., 2011; Yokoo et al., 2007). En grupos de animales cruzados Brahman, por su parte, se obtuvo un PA de $443 \pm 1,7 \mathrm{~kg}$ y GDPA de $1,08 \pm 0,02 \mathrm{~g}$, al ser sometidos a un régimen alimenticio basado en granos y vitaminas (Lunstra y Cundiff, 2003). Los individuos Gucerá registraron un PA de 211,98 kg (Toral et al., 2011). Las diferencias de peso y ganancia de peso presentadas pueden deberse al manejo alimenticio del animal en cada estudio.

En la literatura se reportan valores superiores de P18 para los animales Nelore, de 264 a $347 \mathrm{~kg}$ (Del ValleGarnero et al., 2001; Vieira et al., 2006; Yokoo et al., 2007; Lopes et al., 2010; Gonçalves et al., 2011). Los estudios anteriores fueron realizados en diferentes regiones de la República Federativa de Brasil, por lo que, los pesos inferiores en el presente estudio, pueden deberse a las diferentes condiciones edafoclimáticas entre cada país.

Se registraron P18 superiores a los reportados en el presente estudio para los animales Brahman (363 kg) (Souza-Bertipaglia et al., 2012). Sin embargo, indica que los individuos Brahman pueden tener P18 desde los 111,32 hasta los 540,46 kg, rango dentro del cual se encuentra el P18 observado para estos animales.

En edades posteriores al destete (72 meses), los animales Brahman pueden llegar a presentar pesos entre 500 y $600 \mathrm{~kg}$. Aquellos que son $1 / 2$ Brahman (padre) presentan pesos superiores a los animales Brahman, aunque no son significativamente diferentes ( $\mathrm{P}>0,05)$ (Sandelin et al., 2002). El comportamiento de los individuos con padre Brahman sugiere una mejoría en las características de crecimiento, posteriores al año de edad, sin ser significativamente diferente de otro grupo racial. En otra línea, Sandelin et al. (2002) encontraron que el peso de animales $1 / 2$ Brahman (madre) fue significativamente diferente $(\mathrm{P} \leq 0,05)$ de los animales Brahman y $1 / 2$ Brahman (padre), sin embargo, en el presente estudio no se observaron P18 y GDP18 para animales cruzados hijos de madres Brahman.

El P18 y GDP18 mayor en los animales Brahman, se puede explicar debido a que el grupo racial Brahman se encuentra en el sitio de estudio desde los años setentas por lo que, la superioridad reportada en el presente estudio puede deberse a cuestiones de adaptabilidad de este grupo racial en la zona analizada.

\section{Conclusiones}

En las condiciones analizadas, los factores edafoclimáticos de la zona donde se desarrolló el estudio, influenciaron el comportamiento productivo del animal, por lo que, la atención sobre estas condiciones proporcionará la optimización de los rendimientos.

Los animales machos obtuvieron el mayor peso en edades anteriores al año, en edades posteriores las hembras tendieron a presentar un mayor peso.

Las proporciones crecientes de Nelore proporcionaron mejoramiento en PN y crecimiento al destete, mientras que, proporciones 50\% o 100\% Brahman generaron animales más pesados en edades posteriores al destete. 
Respecto a la influencia de los grupos raciales de los progenitores, sobre las características reportadas por las crías, la raza de la madre influyó en edades tempranas de la cría y el grupo racial del padre en las diferencias posteriores al año de edad.

\section{Agradecimientos}

Los autores agradecen a la Corporación Ganadera, a los propietarios y personal administrativo de la hacienda, por el apoyo brindado para realizar este estudio.

\section{Literatura citada}

Beef Improvement Federation. 2010. Guidelines for uniform beef improvement programs. Beef Improvement Federation, Verona, USA.

Boligon, A.A., L. Galvão-de Albuquerque, M.E. Zerlotti-Mercadante, and R. Barbosa-Lôbo. 2010. Study of relations among age at first calving, average weight gains and weights from weaning to maturity in Nellore cattle. Rev. Bras. Zootec. 39:746751. doi:10.1590/S1516-35982010000400007

Campbell, E.M., J.O. Sanders, D.K. Lunt, C.A. Gill, J.F. Taylor, S.K. Davis, D.G. Riley, and S.B. Smith. 2016. Adiposity, lipogenesis and fatty acid composition of subcutaneous and intramuscular adipose tissues of Brahman and Angus crossbred cattle. J. Anim. Sci. 94:1415-1425. doi:10.2527/jas.2015-9954

Côrtes-Carvalho, G.M., M.N. Lopes-da Frota, A.F. Lima-Neto, D.M.M. Ribeiro-Azevêdo, R.M. de-Araujo-Neto, A. Mellode-Araujo, E. Salas-Pereira, and M.S. de-Souza-Carneiro. 2017. Live weight, carcass, and meat evaluation of Nellore, Curraleiro Pé-Duro, and their crossbred products in Piauí State. Rev. Bras. Zootec. 46:393-399. doi:10.1590/s180692902017000500004

Cubas, A.C., D. Perotto, J.J. dos-Santos-Abrahão, e S.C. Mella. 2001. Desempenho até a desmama de bezerros Nelore e cruzas com Nelore. Rev. Bras. Zootec. 30:694-701. doi:10.1590/S1516-35982001000300013

Dal-Pizzol, J.G., I.P.O. Gomes, W. Braun, J.A.N. Lisboa, K.K.M.C. Flaiban, e A. Thaler-Neto. 2017. Comparação entre vacas puras Holandês e mestiças Holandês x Jersey quanto à sanidade, imunidade e facilidade de parto. Arq. Bras. Med. Vet. Zootec. 69:955-961. doi:10.1590/1678-4162-8867

de-Azambuja-Ribeiro, E.L., J.A. Hernandez, E.L. Zanella, I.Y. Mizubuti, L. da Silva, e J.J. Reeves. 2008. Desempenho e características de carcaça de bovinos de diferentes grupos genéticos. Rev. Bras. Zootec. 37:1669-1673. doi:10.1590/ S1516-3598200800090002

del-Valle-Garnero, A., R. Barbosa-Lôbo, L.A. Framartino-Bezerra, e H. Nunes-de-Oliveira. 2001. Comparação entre alguns critérios de seleção para crescimento na raça nelore. Rev. Bras. Zootec. 30:714-718. doi:10.1590/S151635982001000300016

Dillon, J.A., D.G. Riley, A.D. Herring, J.O. Sanders, and R.M. Thallman. 2015. Genetic effects on birth weight in reciprocal Brahman-Simmental crossbred calves. J. Anim. Sci. 93:553-561. doi:10.2527/jas.2014-8525

FAO. 2015. FAO Statistical pocketbook 2015: World food and agriculture. Job number 14691. FAO, Rome, ITA.

FAO. 2014. Anuario estadístico de la FAO 2014: La alimentación y la agricultura en América Latina y el Caribe. FAO, Santiago, CHI. 
Flôres, A.A., F.E. Madalena, e R.L. Teodoro. 2004. Desempenho comparativo de seis grupos de cruzamento Holandês/Guzerá: 12. ganho de peso de bezerras e novilhas. Rev. Bras. Zootec. 33:1695-1702. doi:10.1590/S1516-35982004000700008

García-Henao, G.A., J.G. Maldonado-Estrada, y J.G. López. 2003. Caracterización productiva y reproductiva de las explotaciones ganaderas del bajo cauca y el litoral atlántico antioqueños. II. Comportamiento de cuatro grupos raciales Bos indicus en un sistema de bosque seco tropical (bs-T). Rev. Col. Cienc. Pecu. 16:117-125.

Gonçalves, F.M., A.V. Pires, I.G. Pereira, D.A. Garcia, M.M. Farah, C.T. Meira, e V.A.R. Cruz. 2011. Avaliação genética para peso corporal em um rebanho Nelore. Arq. Bras. Med. Vet. Zootec. 63:158-164. doi:10.1590/S0102-09352011000100024

IMN (Instituto Meteorológico Nacional). 2008. Atlas interactivo. IMN, CRC. https://www.imn.ac.cr/web/imn/39 (consultado 28 dic. 2017).

INEC (Instituto Nacional de Estadística y Censos). 2014. VI Censo nacional agropecuario INEC, San José, CRC.

Jenkins, T.G., and C.L. Ferrell. 2004. Preweaning efficiency for mature cows of breed crosses from tropically adapted Bos indicus and Bos taurus and unadapted Bos taurus breeds. J. Anim. Sci. 82:1876-1881. doi:10.2527/2004.8261876x

Lopes, J.S., P.R.N. Rorato, T. Weber, R.O. Araújo, M.A. Dornelles, e J.G. Comin. 2010. Avaliação do desempenho na pósdesmama para uma população bovina multirracial Aberdeen Angus x Nelore utilizando-se diferentes modelos genéticos. Arq. Bras. Med. Vet. Zootec. 62:1439-1447. doi:10.1590/S0102-09352010000600021

Lunstra, D.D., and L.V. Cundiff. 2003. Growth and pubertal development in Brahman-, Boran-, Tuli-, Belgian Blue-, Herefordand Angus-sired F1 bulls. J. Anim. Sci. 81:1414-1426. doi:10.2527/2003.8161414x

Marcondes, M.I., L.O. Tedeschi, S.C. Valadares-Filho, and M.L. Chizzotti. 2012. Prediction of physical and chemical body compositions of purebred and crossbred Nellore cattle using the composition of a rib section. J. Anim. Sci. 90:1280-1290. doi:10.2527/jas.2011-3839

Martínez-González, J.C., A. Azuara-Martínez, J. Hernández-Meléndez, G.M. Parra-Bracamonte, y S.P. Castillo-Rodríguez. 2008. Características pre-destete de bovinos simmental (Bos taurus) y sus cruces con brahman (Bos indicus) en el trópico mexicano. Rev. Colomb. Cienc. Pecu. 21:365-371.

McManus, C., R. de-Almeida-Teixeira, L. Talarico-Dias, H. Louvandini, e E.M. Bianchini-Oliveira. 2008. Características produtivas e reprodutivas de vacas Holandesas e mestiças Holandês × Gir no Planalto Central. Rev. Bras. Zootec. 37:819823. doi:10.1590/S1516-35982008000500006

Newman, S.,A. Reverter, and D.J.Johnston. 2002. Purebred-crossbred performance and genetic evaluation of postweaning growth and carcass traits in Bos indicus X Bos taurus crosses in Australia. J. Anim. Sci. 80:1801-1808. doi:10.2527/2002.8071801x.

Perotto, D., J.J. dos-Santos-Abrahão, e I.A. Kroetz. 2006. Intervalo de partos de fêmeas bovinas Nelore, Guzerá x Nelore, Red Angus x Nelore, Marchigiana x Nelore e Simental x Nelore. Rev. Bras. Zootec. 35:733-741. doi:10.1590/S151635982006000300014

Pitombo, R.S., D.D.N. Souza, R.O.S. Ramalho, A.B.A. Figueiredo, V.C. Rodrigues, D.D.G.C. Freitas, e J.C.S. Ferreira. 2013. Qualidade da carne de bovinos superprecoces terminados em confinamento. Arq. Bras. Med. Vet. Zootec. 65:1203-1207. doi:10.1590/S0102-09352013000400036

Ramírez, E.J., M.F. Cerón-Calderón, A.C. Herrera, O.D. Vergara., E.M. Arboleda, y L.F. Restrepo. 2009. Crecimiento de hembras cruzadas en el trópico Colombiano. Rev. Colomb. Cienc. Pecu. 22:642-647.

Rocha-Sarmento, J.L., E.C. Pimenta-Filho, M.N. Ribeiro, e R. Martins-Filho. 2003. Efeitos ambientais e genéticos sobre o ganho em peso diário de bovinos Nelore no Estado da Paraíba. Rev. Bras. Zootec. 32:325-330. doi:10.1590/S151635982003000200010 
Rodrigues, P.F., L.M. Menezes, R.C. Azambuja, R.W. Suñé, I.D. Barbosa-Silveira, and F.F. Cardoso. 2014. Milk yield and composition from Angus and Angus-cross beef cows raised in southern Brazil. J. Anim. Sci. 92:2668-2676. doi:10.2527/ jas.2013-7055

Rodrigues-Paulino, P.V., S. Valadares-Filho, E. Detmann, R. Diniz-Valadares, M. Alves-Fonseca, R.M. Liberal-Véras, e D. Moura-Oliveira. 2008. Desempenho produtivo de bovinos Nelore de diferentes classes sexuais alimentados com dietas contendo dois níveis de oferta de concentrado. Rev. Bras.Zootec. 37:1079-1087. doi:10.1590/S1516-35982008000600019.

Sandelin, B.A., A.H.Jr. Brown, M.A. Brown, Z.B. Johnson, D.W. Kellogg, and A.M. Stelzleni. 2002. Genotype x environmental interaction for mature size and rate of maturing for Angus, Brahman, and reciprocal-cross cows grazing bermudagrass or endophyte infected fescue. J. Anim. Sci. 80:3073-3076. doi:10.2527/2002.80123073x.

SAS Institute. 2009. Base SAS 9.2 procedures guide. SAS Institute Inc., NY, USA.

Schiermiester, L.N., R.M. Thallman, L.A. Kuehn, S.D. Kachman, and M.L. Spangler. 2015. Estimation of breed-specific heterosis effects for birth, weaning, and yearling weight in cattle. J. Anim. Sci. 93:46-52. doi:10.2527/jas.2014-8493

Souza-Bertipaglia, T., L.O. Duitama-Carreño, C.H. Cavallini-Machado, C. Andrighetto, and R. da-Fonseca. 2012. Estimates of genetic parameters for visual scores and their correlation with production and reproductive traits in Brahman cattle. Rev. Bras. Zootec. 41:1407-1411. doi:10.1590/S1516-35982012000600013

SPSS (Statistical Package for the Social Sciences). 2012. IBM SPSS Statistics 21 Core system user' s guide. IBM Corporation, USA.

Teixeira, R.A., e L.G. Albuquerque. 2005. Heteroses materna e individual para ganho de peso pré-desmama em bovinos Nelore $\times$ Hereford e Nelore $\times$ Angus. Arq. Bras. Med. Vet. Zootec. 57:518-523. doi:10.1590/S0102-09352005000400013

Toral, F.R, R.A. Torres-Júnior, P.S. Lopes, e L.O. Campos-da-Silva. 2011. Modelagem de efeitos genéticos e ambientais que influenciam o peso à desmama de bezerros mestiços Charolês-Zebu. Rev. Bras. Zootec. 40:2378-2387. doi:10.1590/ S1516-35982011001100014

Vargas-Leitón, B., y J.J. Romero-Zúñiga. 2010. Efectos genéticos aditivos y no aditivos en cruces rotacionales Holstein $\times$ Jersey y Holstein $\times$ Pardo Suizo. Agron. Mesoam. 21:223-234. doi:10.15517/am.v21i2.4885

Vieira, A., J.F. Piva-Lobato, E. Simöes-Corrêa, R.A. Torres-Júnior, e F. Paim-Costa. 2006. Desenvolvimento e desempenho reprodutivo de novilhas Nelore criadas a pasto nos cerrados do Centro-Oeste brasileiro. Rev. Bras. Zootec. 35:186-192. doi:10.1590/S1516-35982006000100024.

Yokoo, M., L.G. Albuquerque, R. Barbosa-Lôbo, R.D. Sainz, J.M. Carneiro-Júnior, L.A. Framartino-Bezerra, e F. Rodriguesda-Cunha-Araujo. 2007. Estimativas de parâmetros genéticos para altura do posterior, peso e circunferência escrotal em bovinos da raça Nelore. Rev. Bras. Zootec. 36:1761-1768. doi:10.1590/S1516-35982007000800008

Agron. Mesoam. 30(1):195-207, enero-abril, 2019 